\title{
The roles of PTEN expression in gastric cancer: a bibliometric, meta and bioinformatics analysis
}

\author{
Hua-Chuan Zheng ${ }^{1}$, Yu-Hong Qiu ${ }^{1,2}$ and Shuang Zhao ${ }^{1}$ \\ ${ }^{1}$ Department of Experimental Oncology and Animal Center, Shengjing Hospital of China Medical University, Shenyang \\ 110004, China \\ ${ }^{2}$ Library, China Medical University, Shenyang 110004, China
}

Correspondence to: Hua-Chuan Zheng, email: zheng_huachuan@hotmail.com

Keywords: PTEN; gastric cancer; bibliometrics; meta analysis; bioinformatics analysis

Received: August 01, 2017

Accepted: November 03, 2017

Published: January 02, 2018

Copyright: Zheng et al. This is an open-access article distributed under the terms of the Creative Commons Attribution License 3.0 (CC BY 3.0), which permits unrestricted use, distribution, and reproduction in any medium, provided the original author and source are credited.

\section{ABSTRACT}

PTEN encodes a dual phospholipid phosphatase, and is frequently deleted, mutated or down-regulated in a variety of human malignancies. Here, we performed a systematic bibliometric, meta- and bioinformatics analysis through multiple online databases up to March 14, 2017. The co-citation and co-word analysis showed that the study about PTEN and gastric cancer mainly focused on PTEN discovery, correlation of its genetic and epigenetic alteration with cancers, the effects of PTEN expression on the phenotypes of gastric cancer cells, and the regulatory effects of miRNA on PTEN translation. Meta-analysis indicated that down-regulated PTEN expression was seen in gastric cancer in comparison to normal mucosa and dysplasia $(p<0.05)$, and positively with depth of invasion, lymph node and distant metastasis, TNM staging, dedifferentiation and poor prognosis of gastric cancer $(p<0.05)$. According to bioinformatics databases, PTEN mRNA expression was higher in gastric cancer than normal tissues $(p<0.05)$, and positively correlated with depth of invasion and differentiation of gastric cancer $(p<0.05)$. Kaplan-Meier plotter showed that a higher PTEN expression was positively correlated with overall and progression-free survival rates of all cancer patients, even stratified by aggressive parameters $(p<0.05)$. These findings indicated that PTEN expression might be employed as a potential marker to indicate gastric carcinogenesis and subsequent progression, even prognosis.

\section{INTRODUCTION}

PTEN (phosphatase and tensin homology deleted from human chromosome 10, also called as MMAC1 or TEP1) encodes a dual phospholipid phosphatase. It specifically dephosphorylates PIP3 to inhibit Akt signaling pathway, and FAK to suppress cell adhesion, spreading and recognition. It also inhibits she phosphorylation and subsequently Ras/MAP-kinase pathway [1, 2]. PTEN dephosphorylates Akt to suppress membrane GLUT1 expression and glucose consumption in cancer cells [3]. Gu et al. [4] has found that PTEN deficiency resulted in CREB phosphorylation independent of PI3K/Akt pathway. Additionally, Helicobacter pylori might phosphorylate and inactivate PTEN at Ser380/ Thr382/383 to promote gastric epithelial cell survival via PI3K/Akt pathway
[5]. CK2 kinase phosphorylates ser/thr residues of PTEN for its stability due to proteasomal degradation resistance [6]. PTEN $\alpha$ and PTEN $\beta$ are N-terminally extended forms of PTEN and initiated from CUG and AUU codons upstream of the coding region of canonical PTEN. PTEN $\alpha$ can up-regulate cytochrome c oxidase activity and ATP synthesis, and PTEN $\beta$ physically associates with and dephosphorylates nucleoli to suppress ribosomal biogenesis $[7,8]$. PTEN interacts with DAXX, and subsequently modulateds oncogene expression via diassociation of DAXX-H3.3 on the chromatin [9]. For example, PTEN induces transcriptional activity of HIF-2 $\alpha$ by suppressing the expression of Yin Yang 1 via PI3K/Akt pathway [10].

MKRN1 E3 ligase may enhance EGFR/PI3K/Aktmediated ubiquitination and subsequent degradation of 
PTEN protein [11]. PTEN mono-ubiquitination promotes protein stability and nuclear localization [12], where PTEN interferes with interaction of Ku70 with doublestrand breaks via post-translational poly (ADP-ribosyl) ation of PARP1 [13]. HECT E3-ligase NEDD4-1 is a proto-oncogenic ubiquitin ligase to mediate proteasomal degradation of PTEN [14, 15]. NEDD4-like protein family, WWP2, physically interacts with PTEN and mediates PTEN degradation through an ubiquitylationdependent pathway [16]. However, the inhibitory effects of PTEN on NEDD4 expression are diminished by a mutation (C124S) in the catalytic site of PTEN [17]. Deubiquitylation and stabilization of PTEN by USP13 suppresses tumorigenesis and glycolysis in PTENpositive breast cancer cells [18]. Ret finger protein can promote atypical polyubiquitination of PTEN to inhibit PTEN phosphatase activity [19]. K163 acetylation and activation of PTEN is mediated by HDAC6 inhibition, which suppresses carcinogenesis and subsequent progression [20].

$P T E N$, as an important tumor suppressor gene, is frequently deleted, mutated or down-regulated in various malignancies [1] and its conditional abrogation of PTEN resulted in organ-specific carcinogenesis, including hepatocellular cancer, urothelial carcinoma, squamous cell carcinoma of vagina and rectum, colonic adenocarcinoma, prostate cancer, papillomas, squamous cell carcinomas and T-cell lymphoma [21]. In the present study, we tried to map the history, emerging trends and research front of PTEN and gastric cancer using CiteSpace II. After that, we investigated the roles of PTEN expression in gastric cancer at both mRNA and protein levels by a meta- and bioinformatics analysis.

\section{RESULTS}

\section{The research history, current and future hotspots of PTEN in gastric cancer}

CiteSpace II could help us to facilitate the understanding and interpretation of structural and temporal network patterns. Here, we established a network (Figure 1A) using co-citation analysis as shown in Tables 1-3. The highly-cited, centered and burst articles indicated that PTEN discovery, correlation of its genetic and epigenetic alteration with cancers and the regulatory effects of miRNA on PTEN translation would be history, current situation and future hotpots about the roles of PTEN in gastric cancer. Among them, the papers whose titles contain "PTEN" should be read and investigated. On the other hand, we established a network of the key words from 597 articles (Figure 1B). The top frequent key and burst words indicated that investigators mainly explored the relationship between PTEN and gastric carcinogenesis, its effects on phenotypes of gastric cancer cells, and the correlation between PTEN and miRNA (Tables 4 and 5).

\section{Selection and characteristics of preferable studies}

We selected 17 articles paper for our meta-analysis as shown in Figure 2 and Table 6. Only 5 articles include gastric normal mucosa and cancer samples [22-26], 3 do gastric dysplasia and cancer samples [23-25], 16 do the relationship between PTEN expression and clinicopathological features of gastric cancer [22-37] and 6 do survival data about PTEN expression [25, 28, 30, 33, 36, 38].

\section{The clinicopathological and prognostic significances of PTEN expression in gastric cancer}

In 5 studies, 663 cancers and 361 controls were involved in our analysis. PTEN expression was lower in gastric cancer than normal mucosa epithelium (Figure $3 \mathrm{~A}, p<0.00001)$. Cancer susceptibility was the same for the PTEN-negative dysplasia using 483 cancers and 103 dysplasia (Figure 3B, $p<0.00001$ ). There was a higher PTEN expression in female than male patients with gastric cancer (Figure 3C, $p=0.03$ ). PTEN expression was inversely linked to depth of invasion (Figures 3D and 3E, $p<0.05$ ), lymph node metastasis (Figure 3F, $p<0.00001$ ), distant metastasis (Figure 3G, $p<0.00001$ ), TNM staging (Figures 3H and 3I, $p<0.01$ ), dedifferentiation (Figure 3J, $p=0.01$ ) and un favorable prognosis (Figure $3 \mathrm{~K}, \mathrm{HR}=$ 2.34, 95\% CI: 1.85-2.96, $p<0.00001)$ of gastric cancer.

\section{Heterogeneity analysis}

To assess the heterogeneity, we deleted a study to observe the alteration in the pooled results of remaining studies (Figure 4). For example, the pooled OR about relationship between PTEN expression and gender was remarkably decreased if Zheng (2007)'s study was excluded (data not shown).

\section{The clinicopathological and prognostic significances of PTEN mRNA expression in gastric cancer}

DErrico's and Wang's datasets were used to perform bioinformatics analysis. PTEN mRNA expression was found to be higher in gastric cancer than normal tissues, even stratified into intestinal-, diffuse- and mixed-type carcinomas (Figure 5A, $p<0.05$ ). TCGA data showed that PTEN mRNA expression was positively correlated with depth of invasion (Figure 5B, $p<0.05$ ), histological grading (Figure 5B, $p<0.05$ ) and dedifferentiation (Figure $5 \mathrm{~B}, p<0.05)$ of gastric cancers. Kaplan-Meier plotter showed a positive correlation between PTEN mRNA expression and overall or progression-free survival rate of all, T2, T3, N1-3, N1, N2, M0, moderately-differentiated, 
A

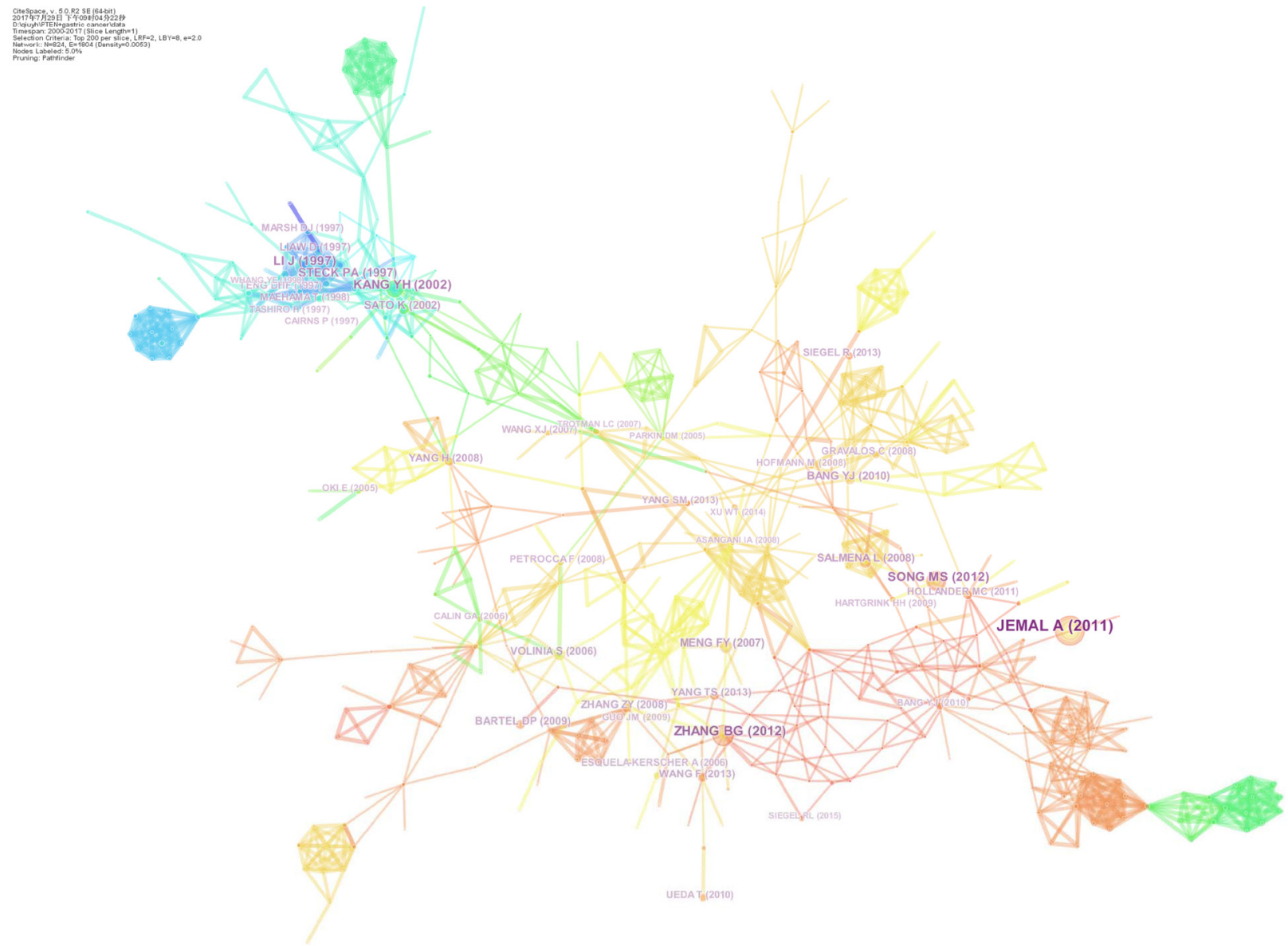

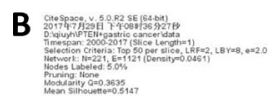

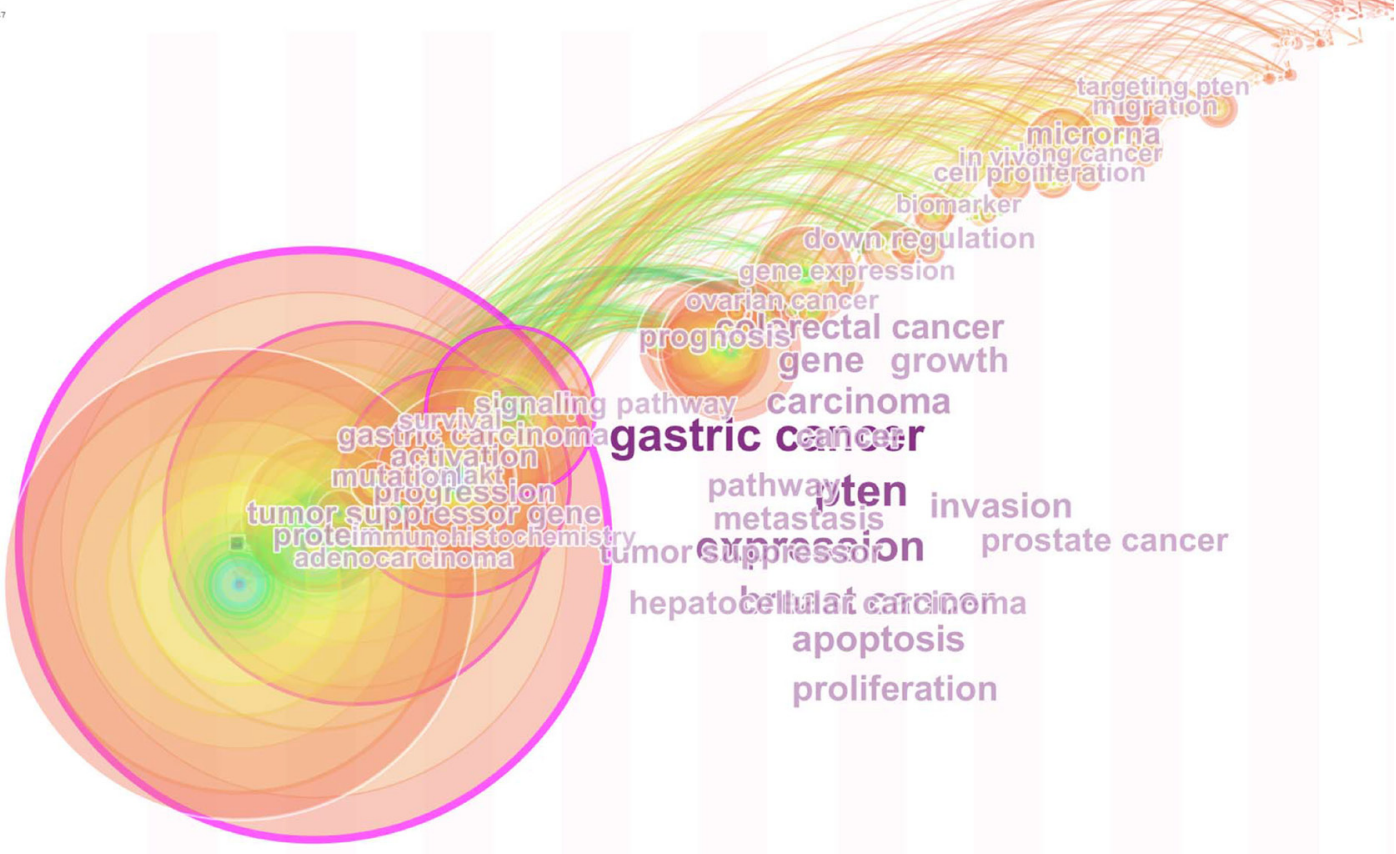

Figure 1: The cluster analysis of co-cited references. (A) and highly-frequent key words (B). 
Table 1: The top 10 highly-cited articles

\begin{tabular}{|c|c|c|c|c|c|}
\hline Rank & Freq & Author & Year & Source & Title \\
\hline 1 & 41 & Jemal A & 2011 & CA-Cancer J Clin & Global cancer statistics. \\
\hline 2 & 28 & Zhang BG & 2012 & Oncol Rep & $\begin{array}{l}\text { microRNA- } 21 \text { promotes tumor proliferation and invasion in gastric } \\
\text { cancer by targeting PTEN. }\end{array}$ \\
\hline 3 & 26 & Song MS & 2012 & Nat Rev Mol Cell Bio & The functions and regulation of the PTEN tumour suppressor. \\
\hline 4 & 24 & Kang YH & 2002 & Lab Invest & Promoter methylation and silencing of PTEN in gastric carcinoma. \\
\hline 5 & 23 & Li J & 1997 & Science & $\begin{array}{l}\text { PTEN, a putative protein tyrosine phosphatase gene mutated in human } \\
\text { brain, breast, and prostate cancer. }\end{array}$ \\
\hline 6 & 20 & Steck PA & 1997 & Nat Genet & $\begin{array}{l}\text { Identification of a candidate tumour suppressor gene, MMAC1, at } \\
\text { chromosome } 10 \mathrm{q} 23.3 \text { that is mutated in multiple advanced cancers. }\end{array}$ \\
\hline 7 & 16 & Meng FY & 2007 & Gastroenterology & $\begin{array}{l}\text { MicroRNA-21 regulates expression of the PTEN tumor suppressor } \\
\text { gene in human hepatocellular cancer. }\end{array}$ \\
\hline 8 & 15 & Salmena L & 2008 & Cell & Tenets of PTEN tumor suppression. \\
\hline 9 & 15 & Sato K & 2002 & Virchows Arch & $\begin{array}{l}\text { Analysis of genetic and epigenetic alterations of the PTEN gene in } \\
\text { gastric cancer. }\end{array}$ \\
\hline 10 & 15 & Bang YJ & 2010 & Lancet & $\begin{array}{l}\text { Trastuzumab in combination with chemotherapy versus chemotherapy } \\
\text { alone for treatment of HER2-positive advanced gastric or gastro- } \\
\text { oesophageal junction cancer (ToGA): a phase 3, open-label, randomised } \\
\text { controlled trial. }\end{array}$ \\
\hline
\end{tabular}

stage III, intestinal-type or Her2-positivve cancer patients (Figure $5 \mathrm{C}$ and Table 7, $p<0.05$ ). Stage I or IV, mixedtype or Her2-negative cancer patients with PTEN mRNA hyperexpression showed a favorable overall prognosis than those with its hypoexpression (Table $7, p<0.05$ ). There was a positive correlation of PTEN expression with a progression-free survival rate of stage II cancer patients (Table 7, $p<0.05$ ).

\section{DISCUSSION}

PTEN induces cell cycle arrest by inhibition of Notch signaling [39], and suppresses invasion through repression of epithelial-mesenchymal transition [40] and paxillin transcription via $\mathrm{PI} 3 \mathrm{~K} / \mathrm{Akt} / \mathrm{NF}-\kappa \mathrm{B}$ pathway [41]. PTEN overexpression suppresses adhesion, invasion and metastasis in osteosarcoma and gastric

\section{Literature identified from database \\ Pubmed: 248 records \\ Web of Science: 597 records \\ BIOSIS: 507 records \\ SciFinder: 765 records}

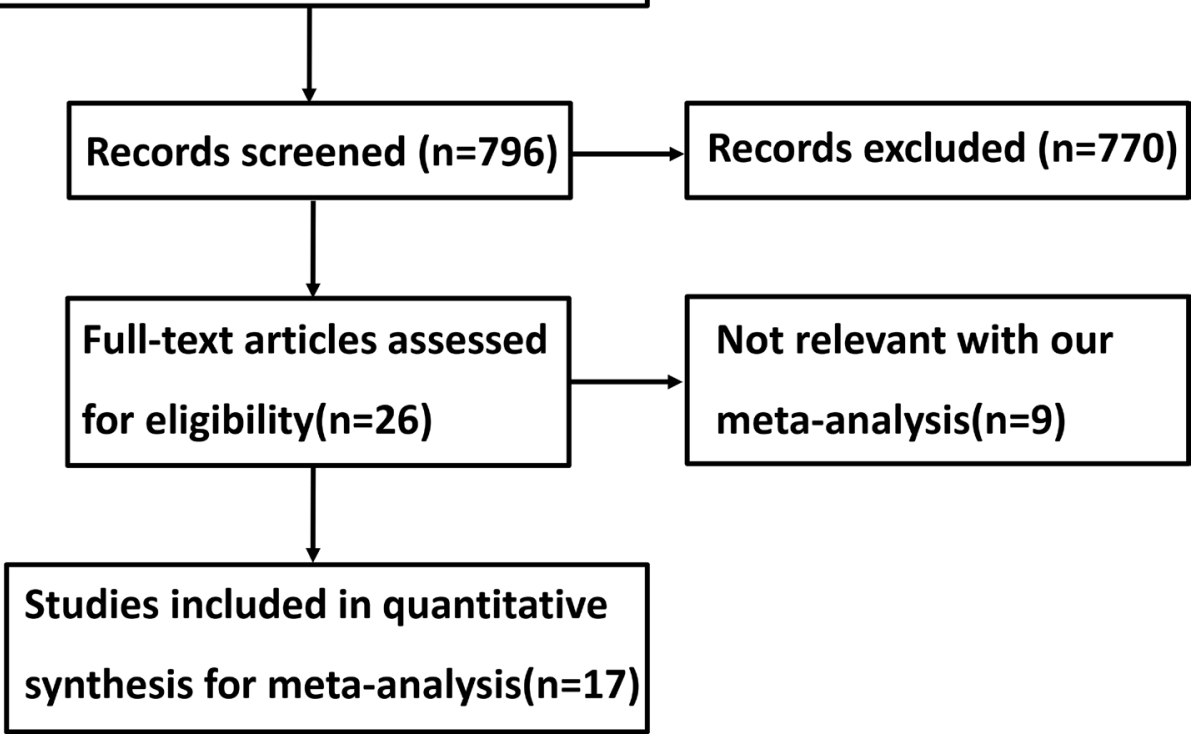

Figure 2: Flow diagram of the selection process in this meta-analysis. 


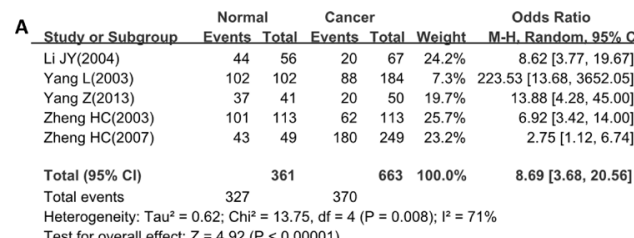

烈

$\begin{array}{llll}\text { B } & \begin{array}{c}\text { Dysplasia } \\ \text { Events Total }\end{array} & \text { Cancer } & \text { Odds Ratio } \\ \text { Study or Subgroup Total Weight } & \text { M-H. Fixed. 95\% C }\end{array}$ Odds Ratio

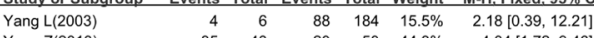
$\begin{array}{llll}20 & 50 & 44.3 \% & 4.04[1.72,9.4\end{array}$

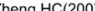

Total $(95 \% \mathrm{Cl})$

Total events $\quad 84 \quad 288$ Heterogeneity: Chi $=0.47, \mathrm{df}=2(P=0.79)$
Test for overall effect: $Z=4.24(P<0.0001)$

$483 \quad 100.0 \% \quad 3.86[2.07,7.21]$

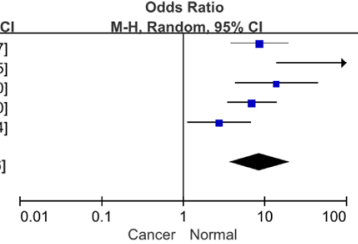

$\begin{array}{ccc}\text { Male } & \begin{array}{c}\text { Female } \\ \text { Study or Subgroup }\end{array} & \begin{array}{c}\text { Odds Ratio } \\ \text { Events }\end{array} \text { Total Events Total Weight } \\ \text { M-H. Fixed. 95\% }\end{array}$

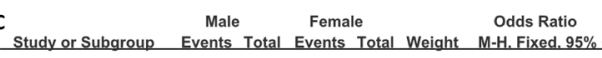
\begin{tabular}{llllllll}
\hline Badary DM(2017) & 13 & 26 & 10 & 16 & $4.3 \%$ & $0.60[0.17,2.14]$ \\
\hline & 49 & 71 & 16 & 20 & $5.7 \%$ & $0.52[0.16,1.74]$
\end{tabular} Bai ZG(2007)

Deng $\mathrm{H}(2006)$

Kang $\mathrm{HJ}(2016)$

Kim HS(2016)

Li Y(2013)

Yang Z(2013)

Zhang XL(2015)

Zheng HC(2007)
Zhu XH(2013)

$\begin{array}{lrllll}59 & 82 & 31 & 38 & 8.3 \% & 0.58[0.22,1.50]\end{array}$

$\begin{array}{lllll}129 & 203 & 27 & 31 & 12.0 \%\end{array}$

$\begin{array}{rrrrr}231 & 300 & 118 & 138 & 26.1 \% \\ 7 & 15 & 11 & 18 & 3.7 \%\end{array}$

$0.26[0.09,0.77]$

$0.57[0.33,0.98]$

$0.56[0.14,2.23]$

$1.23[0.56,2.68]$

$\begin{array}{rrrrr}31 & 72 & 16 & 42 & 8.1 \% \\ 8 & 23 & 9 & 20 & 4.4 \%\end{array}$

$\begin{array}{rrrrrr}8 & 23 & 9 & 20 & 4.4 \% & 0.65[0.19,2.23] \\ 21 & 40 & 4 & 8 & 2.2 \% & 1.11[0.24,505]\end{array}$

Total $(95 \% \mathrm{Cl})$

$\begin{array}{lcc}\text { Total events } & 729 & 312 \\ \text { Heterogeneity: } C h i^{2}=11.96, d f=10 & (P=0.29) ; i^{2}=16 \%\end{array}$

$\begin{array}{lll}46 & 72 & 13.7 \% \\ 24 & 47 & 11.4 \%\end{array}$

$1.32[0.74,2.36]$

Test for overall effect: $Z=2.18(P=0.03)$

$\begin{array}{cccc}\text { TO }-1 & \text { T2-4 } & \text { Odds Ratio } \\ \text { Study or Subgroup } & \text { Events Total Events Total Weight } & \text { M-H. Random. } 95\end{array}$

$400.0 \% \quad 0.76[0.59,0.97]$

Bai ZG(2007)

Deng $\mathrm{H}(2006)$

Im SA(2005)

Kang $\mathrm{H} J(2010$

Li JY(2004)

Tapia O(2014)

Yang L(2003)

Yang Z(2013)

Zheng HC(2003)

Zhu XH(2013)

$\begin{array}{lllll}5 & 4 & 19 & 38 & 2.7 \% \\ 5 & 6 & 59 & 85 & 4.4 \%\end{array}$

$9.00[0.45,178.69]$

$\begin{array}{rrrr}9 & 38 & 2.7 \% & 9.00[0.45,178.69] \\ 9 & 85 & 4.4 \% & 2.20[0.25,19.81] \\ 5 & 107 & 8.4 \% & 0.22[0.06,0.77] \\ 29 & 61 & 10.5 \% & 4.10[1.55,10.86\end{array}$

$\begin{array}{rrr}85 & 107 & 8.4 \% \\ 29 & 61 & 10.5 \%\end{array}$

$4.10[1.55,10.86]$

$3.21[1.77,5.84]$
$0.66[0.17,2.51]$

26
26 $8.1 \%$

4211

$\begin{array}{lll}26 & 56 & 8.1 \% \\ 12 & 27 & 2.8 \%\end{array}$

$0.66[0.17,2.51]$

$6.12[0.83,314.64]$

$\begin{array}{rr}29 & 71 \\ 63 & 147\end{array}$

Total $(95 \% \mathrm{Cl})$

$\begin{array}{lcc}\text { Total events } & 294 & 600 \\ \text { Heterogeneity: } \text { Tau }^{2}=0.49 ; \mathrm{Ch}^{2}=29.79, & \mathrm{df}=11(P=0.002) ;\left.\right|^{2}=63 \%\end{array}$

Not estimable
$2.78[1.30,5.95]$

$0.56[0.10,3.28]$

$2.78[1.06,7.28]$

$5.09[2.70,9.59]$
$1.70[0.48,6.07]$

$\begin{array}{lll}71 & 124 & 12.9 \% \\ 75 & 148 & 8.5 \%\end{array}$

$2.14[1.24,3.70]$ Test for overall effect: $Z=2.74(P=0.006)$

$\mathbf{E}$

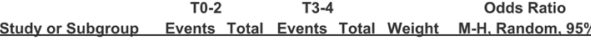

$\begin{array}{lllllll}\text { Badary DM(2017) } & 13 & 13 & 10 & 29 & 2.9 \% & 50.14[2.70,930.34]\end{array}$

$\begin{array}{lllllrr}\text { Bai ZG(2007) } & 16 & 18 & 48 & 73 & 7.4 \% & 4.17[0.89,19.58] \\ \text { Deng H(2006) } & 19 & 29 & 71 & 89 & 12.0 \% & 0.48[0.19,1.21]\end{array}$

Deng $\mathrm{H}(2006)$

Kang $\mathrm{HJ}(2016)$

Li Y(2013)

Tapia $\mathrm{O}(2014)$

Yang Z(2013)

Zheng $\mathrm{HC}(2003)$

Zhu XH(2013)

Total $(95 \% \mathrm{Cl}$

$\begin{array}{rrrrr}36 & 46 & 15 & 38 & 11.7 \%\end{array}$

$\begin{array}{rrrrr}110 & 154 & 46 & 80 & 15.4 \% \\ 14 & 26 & 33 & 88 & 12.4 \%\end{array}$

$\begin{array}{rrrrr}14 & 26 & 33 & 88 & 12.4 \% \\ 3 & 4 & 26 & 67 & 4.2 \%\end{array}$

$\begin{array}{rrrrr}3 & 4 & 26 & 67 & 4.2 \% \\ 3 & 11 & 14 & 32 & 7.6 \%\end{array}$

$\begin{array}{rrrrr}39 & 60 & 23 & 53 & 13.5 \% \\ 17 & 28 & 68 & 134 & 12.9 \%\end{array}$

$683100.0 \%$

Heterogeneity: $\mathrm{Tau}^{2}=0.42 ; \mathrm{Ch}^{2}=23.78, \mathrm{df}=9(\mathrm{P}=0.005) ; \mathrm{I}^{2}=62 \%$ Test for overall effect $Z=2.49(P=0.01)$

$$
\text { LN- }
$$

$$
\mathrm{df}=9(\mathrm{P}+\mathrm{C}+
$$
$0.48[0.19,1.21]$ $5.52[2.12,14.36]$ $1.85[1.05,3.25]$
$1.94[0.80,4.70]$ $1.94[0.80,4.70]$ $4.73[0.47,47.94]$ $0.48[0.11,2.16]$
$2.42[1.13,5.18]$ $2.42[1.13,5.18$ $1.50[0.65,3.44]$

$1.99[1.16,3.43]$

Study or Subgroup Events Total Events Total Weight M-H. Random $95 \%$

\begin{tabular}{lrrrrrr} 
Study or Subgroup & Events & Total & Events & Total & Weight & M-H. Random. 95\% C \\
\hline Badary DM(2017) & 9 & 14 & 19 & 28 & $5.2 \%$ & $0.85[0.22,3.29]$ \\
\hline
\end{tabular}

Im SA(2005)

Li JY(2004)

Li M(2012)

Tapia O(2014)

Yang L(2003)
Yang Z(2013)

Zheng HC(2007)

$\begin{array}{rr}9 & 14 \\ 23 & 27\end{array}$

$3.23[0.99,10.48]$
$1.58[0.54,4.64]$

$2.50[1.01,6.16]$

$4.00[2.72,5.87]$

$9.00[2.17,37.38]$

$32.50[4.68,225.53]$

$1.74[0.80,3.80]$

$5.16[1.61,16.52$

$2.56[1.35,4.83]$

$2.10[0.48,9.17]$

$4.38[2.42,7.94]$

$1.06[0.51,2.24]$

Total $(95 \% \mathrm{Cl})$

Total events

Heterogeneity: Tay $=0.490 \quad 458$

Test for 0 .

D DM- $\quad$ Odds Ratio

Study or Subgroup Events Total Events Total Weight M-H. Fixed, 95\% C $\begin{array}{llllllll}\text { Badary DM(2017) } & 12 & 17 & 11 & 25 & 11.9 \% & 3.05[0.83,11.30]\end{array}$

Bai ZG(2007)

Im SA(2005)

Zheng HC(2007)

Total $(95 \%$ Cl)

Total events 407

Test for overall effect: $Z=4.61(P<0.00001)$

$\begin{array}{rrrr}11 & 25 & 11.9 \% & 3.05[0.83,11.30] \\ 12 & 20 & 22.8 \% & 1.82[0.65,5.15]\end{array}$

$\begin{array}{rrrr}9 & 20 & 24.4 \% & 2.01[0.74,5.45] \\ 2 & 22 & 7.5 \% & 9.57[2.12,43.34]\end{array}$

$\begin{array}{llll}2 & 22 & 7.5 \% & 9.57[2.12,43.34] \\ 4 & 15 & 8.5 \% & 8.34[256,27.22]\end{array}$

$\begin{array}{rrr}11 & 24.8 \% & 0.88[0.26,3.01]\end{array}$

$113 \quad 100.0 \% \quad 2.92[1.85,4.60]$

$r^{2}=52 \%$

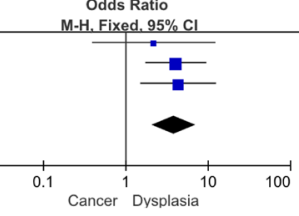

Odds M-H. Fixed. $95 \%$ C
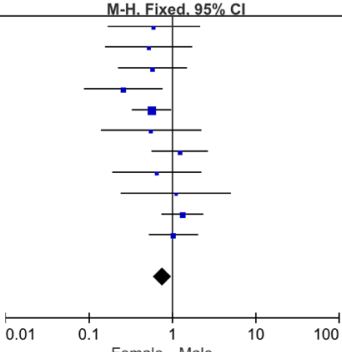

Female Male
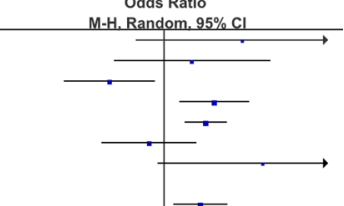

70]

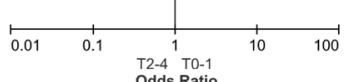

Odds Ratio

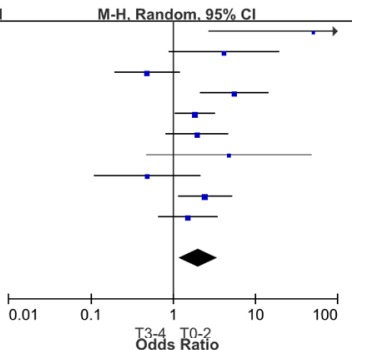

M-H. Odds Ratio

]
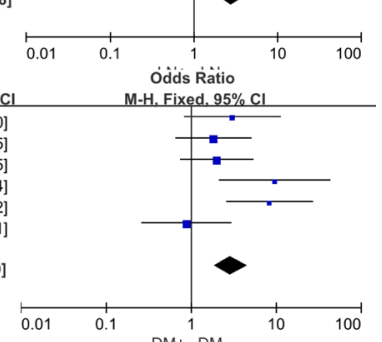


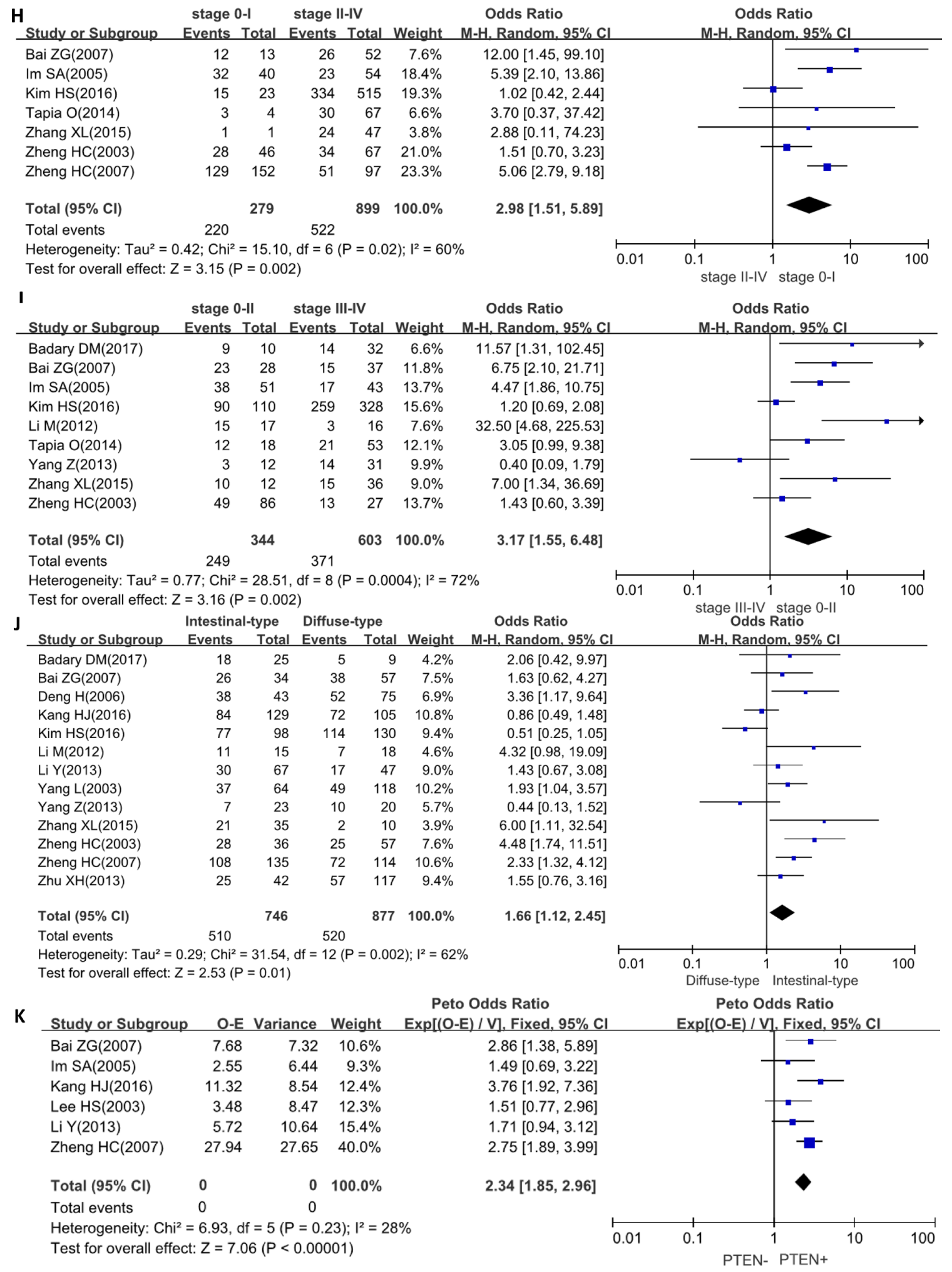

Figure 3: Forest plot for the relationship between PTEN expression and clinicopatholoiocal parameters of gastric cancer. (A) gastric carcinogenesis (cancer $v s$ normal mucosa); (B) gastric carcinogenesis (cancer $v s$ dysplasia); (C) correlation between sex and PTEN expression (male $v s$ female); (D) correlation between depth of invasion and PTEN expression (T0-1 vs T2-4); (E) correlation between depth of invasion and PTEN expression (T0-2 vs T3-4); (F) correlation between lymph node metastasis (LN) and PTEN expression ( $\mathrm{LN}-v s \mathrm{LN}+)$; (G) correlation between distant metastasis (DM) and PTEN expression (DM- $v s \mathrm{DM}+)$; (H) correlation between TNM staging and PTEN expression (stage 0-I vs II-IV); (I) correlation between TNM staging and PTEN expression (stage 0 -II vs III-IV); (J) correlation between differentiation and PTEN expression (intestinal-type vs diffuse-type); (K) correlation between prognosis and PTEN expression (PTEN- vs PTEN+). 
cancer cells with down-regulation of MMP-9, FAK and p-FAK [42, 43]. PTEN inhibits PI3K/NF- $\kappa$ B pathway and the binding of NF- $\kappa$ B to FAK promoter [43]. Loss of PTEN induces tubulin- based microtentacles for migration and metastasis through PI3K-independent activation of cofilin [44]. PTEN suppresses hyaluronic acid-induced MMP-9 expression in glioblastoma cells via FAK dephosphorylation [45]. Nuclear PTEN arrests cell cycle by suppressing cyclin D1 transcription, which is weakened by ERK1/2 activation [46]. In cancer cells, DNA-damaging agents results in ATM-mediated PTEN phosphorylation and the nuclear translocation PTEN to induce autophagy [47]. PTEN was reported to enhance autophagy by inhibiting ubiquitin-proteasome and PI3K/ Akt pathways respectively $[48,49]$. Here, our bibliometric analysis indicated that the investigators mainly focused on PTEN discovery, correlation of its genetic and epigenetic alteration with cancers and the regulatory effects of miRNA on PTEN translation in the roles of PTEN expression in gastric cancer. Therefore, we performed a meta- and bioinformatics analysis about PTEN expression in gastric cancer at both mRNA and protein levels in the following work.

Gastric precancerous lesions appear between gastric epithelium and adenocarcinoma, and are divided into adenomatous, regenerative, crysptal and globoid dysplasia [50]. Jang et al. [51] reported that nuclear PTEN expression was gradually down-regulated during colorectal mucosa-adenoma-adenocarcinomametastasis sequence. Consistent with the data about
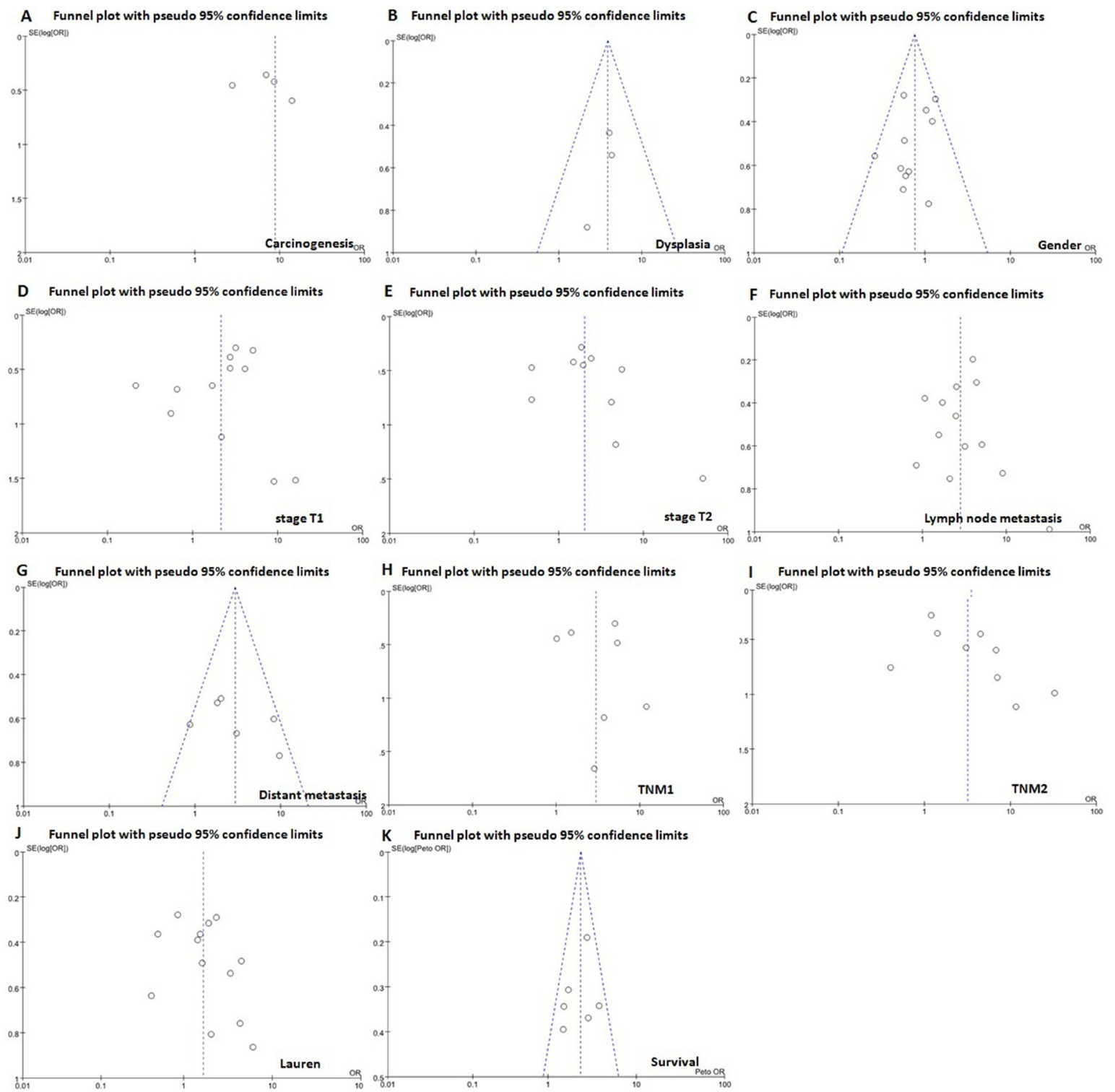

Figure 4: Funnel plot for publication bias test between PTEN expression and gastric carcinogenesis or progression. The bias was analyzed about risk degrees of PTEN expression in gastric mucosa. (A) and dysplasia (B) for gastric carcinogenesis. Additionally, it was tested between PTEN expression and clinicopathological features of gastric cancer, including age (C), depth of invasion (D-E), lymph node metastasis $(\mathbf{F})$, distant metastasis $(\mathbf{G})$, TNM staging $(\mathbf{H}-\mathbf{I})$, and differentiation $(\mathbf{J})$ and prognosis $(\mathbf{K})$. 
Table 2: The top 10 centered articles

\begin{tabular}{|c|c|c|c|c|c|c|}
\hline Rank & Freq & Centrality & Author & Year & Source & Title \\
\hline 1 & 14 & 0.13 & Yang H & 2008 & Cancer Res & $\begin{array}{l}\text { MicroRNA expression profiling in human ovarian cancer: miR- } \\
214 \text { induces cell survival and cisplatin resistance by targeting } \\
\text { PTEN. }\end{array}$ \\
\hline 2 & 8 & 0.12 & Trotman LC & 2007 & Cell & $\begin{array}{l}\text { Ubiquitination regulates PTEN nuclear import and tumor } \\
\text { suppression. }\end{array}$ \\
\hline 3 & 5 & 0.12 & Barbi S & 2010 & $\begin{array}{l}\text { J Exp Clin } \\
\text { Cancer Res }\end{array}$ & $\begin{array}{l}\text { The analysis of PIK } 3 \text { CA mutations in gastric carcinoma and } \\
\text { metanalysis of literature suggest that exon-selectivity is a signature } \\
\text { of cancer type. }\end{array}$ \\
\hline 4 & 24 & 0.11 & Kang YH & 2002 & Lab Invest & Promoter methylation and silencing of PTEN in gastric carcinoma. \\
\hline 5 & 12 & 0.11 & $\begin{array}{l}\text { Hollander } \\
\text { MC }\end{array}$ & 2011 & Nat Rev Cancer & $\begin{array}{l}\text { PTEN loss in the continuum of common cancers, rare syndromes } \\
\text { and mouse models. }\end{array}$ \\
\hline 6 & 8 & 0.11 & Asangani IA & 2008 & Oncogene & $\begin{array}{l}\text { MicroRNA-21 (miR-21) post- transcriptionally downregulates } \\
\text { tumor suppressor Pded } 4 \text { and stimulates invasion, intravasation } \\
\text { and metastasis in colorectal cancer. }\end{array}$ \\
\hline 7 & 4 & 0.09 & Lee HS & 2003 & J Pathol & $\begin{array}{l}\text { Tumour suppressor gene expression correlates with gastric cancer } \\
\text { prognosis. }\end{array}$ \\
\hline 8 & 7 & 0.08 & Tan MH & 2012 & $\begin{array}{l}\text { Clin Cancer } \\
\text { Res }\end{array}$ & $\begin{array}{l}\text { Lifetime cancer risks in individuals with germline PTEN } \\
\text { mutations. }\end{array}$ \\
\hline 9 & 6 & 0.08 & Ambs $S$ & 2008 & Cancer Res & $\begin{array}{l}\text { Genomic profiling of microRNA and messenger RNA reveals } \\
\text { deregulated microRNA expression in prostate cancer. }\end{array}$ \\
\hline 10 & 5 & 0.07 & Deng NT & 2012 & Gut & $\begin{array}{l}\text { Discovery of potential piRNAs from next generation sequences of } \\
\text { the sexually mature porcine testes. }\end{array}$ \\
\hline
\end{tabular}
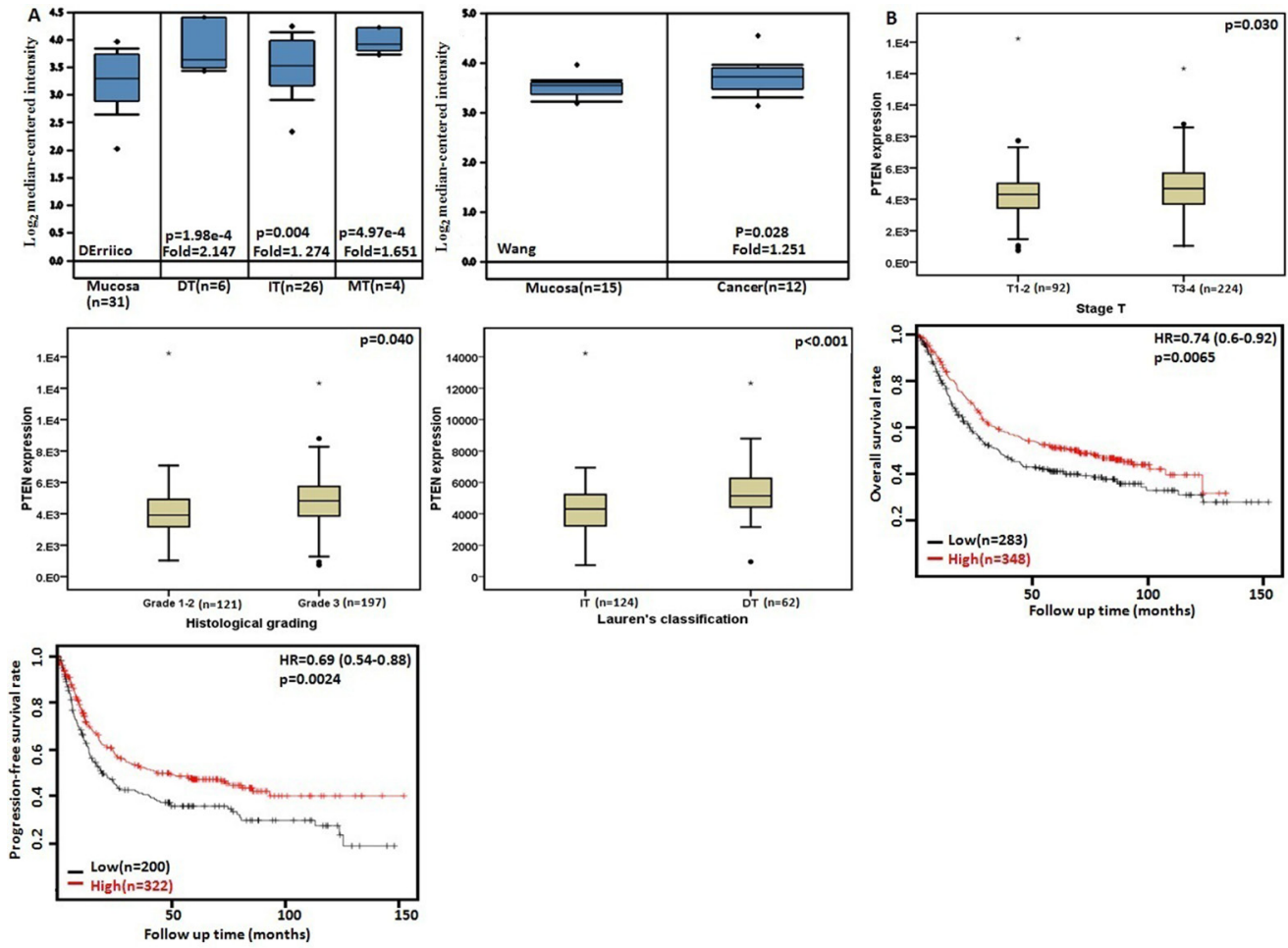

Figure 5: PTEN mRNA expression in gastric carcinogenesis and subsequent progression. DErrico's and Wang's datasets were employed for bioinformatics analysis to analyze PTEN expression during gastric carcinogenesis. (A) higher PTEN expression was detectable in gastric cancer than that in normal gastric mucosa, even stratified into intestinal (IT)-, diffuse-(DT), and mixed-type (MT) carcinomas by Lauren's classification (A, $p<0.05$ ). TCGA database shows that PTEN was more expressed in T3-T4 than T1-T2 cancers (B) $(p<0.05)$. PTEN expression was positively correlated with histological grading and differentiation of gastric cancers $(\mathrm{B}, p<0.05)$. According to the data from Kaplan-Meier plotter, PTEN expression was positively related to both overall and progression-free survival rates of the patients with gastric cancer $(\mathbf{C})$. HR, hazard ratio. 
Table 3: The top 10 burst citation articles

\begin{tabular}{|c|c|c|c|c|c|c|}
\hline Rank & Freq & Centrality & Author & Year & Source & Title \\
\hline 1 & 23 & 11.73 & Li J & 1997 & Science & $\begin{array}{l}\text { PTEN, a putative protein tyrosine phosphatase gene mutated in human } \\
\text { brain, breast, and prostate cancer. }\end{array}$ \\
\hline 2 & 24 & 10.7 & Kang YH & 2002 & Lab Invest & Promoter methylation and silencing of PTEN in gastric carcinoma. \\
\hline 3 & 20 & 10.19 & Steck PA & 1997 & Nat Genet & $\begin{array}{l}\text { Identification of a candidate tumour suppressor gene, MMAC1, at } \\
\text { chromosome 10q23.3 that is mutated in multiple advanced cancers. }\end{array}$ \\
\hline 4 & 15 & 6.65 & Sato K & 2002 & Virchows Arch & $\begin{array}{l}\text { Analysis of genetic and epigenetic alterations of the PTEN gene in } \\
\text { gastric cancer. }\end{array}$ \\
\hline 5 & 13 & 6.56 & Liaw D & 1997 & Nat Genet & $\begin{array}{l}\text { Germline mutations of the PTEN gene in Cowden disease, an inherited } \\
\text { breast and thyroid cancer syndrome. }\end{array}$ \\
\hline 6 & 11 & 5.93 & Maehama T & 1998 & J Biol Chem & $\begin{array}{l}\text { The tumor suppressor, PTEN/MMAC1, dephosphorylates the lipid } \\
\text { second messenger, phosphatidylinositol 3,4,5- trisphosphate. }\end{array}$ \\
\hline 7 & 11 & 5.55 & Marsh DJ & 1997 & Nat Genet & $\begin{array}{l}\text { Germline mutations in PTEN are present in Bannayan-Zonana } \\
\text { syndrome. }\end{array}$ \\
\hline 8 & 10 & 5.26 & Teng DHF & 1997 & Cancer Res & $\begin{array}{l}\text { MMAC1/PTEN mutations in primary tumor specimens and tumor cell } \\
\text { lines. }\end{array}$ \\
\hline 9 & 9 & 4.73 & Cairns P & 1997 & Cancer Res & Frequent inactivation of PTEN/MMAC1 in primary prostate cancer. \\
\hline 10 & 9 & 4.73 & Tashiro H & 1997 & Cancer Res & $\begin{array}{l}\text { Mutations in PTEN are frequent in endometrial carcinoma but rare in } \\
\text { other common gynecological malignancies. }\end{array}$ \\
\hline
\end{tabular}

Table 4: The top 20 key words

\begin{tabular}{llllll}
\hline Rank & Freq & Keyword & Rank & Freq & Keyword \\
\hline 1 & 256 & gastric cancer & 11 & 56 & growth \\
2 & 209 & pten & 12 & 52 & prostate cancer \\
3 & 168 & expression & 13 & 51 & pathway \\
4 & 104 & breast cancer & 14 & 50 & tumor suppressor \\
5 & 79 & carcinoma & 15 & 50 & metastasis \\
6 & 75 & apoptosis & 16 & 46 & cancer \\
7 & 74 & gene & 17 & 44 & hepatocellular carcinoma \\
8 & 69 & colorectal cancer & 18 & 42 & cell \\
9 & 64 & proliferation & 19 & 40 & tumor suppressor gene \\
10 & 57 & invasion & 20 & 40 & activation \\
\hline
\end{tabular}

Table 5: The top 20 burst key words

\begin{tabular}{llllllll}
\hline Rank & Freq & Burst & Keyword & Rank & Freq & Burst & Keyword \\
\hline 1 & 40 & 9.65 & tumor suppressor gene & 11 & 9 & 3.83 & her2 \\
2 & 34 & 7.33 & gastric carcinoma & 12 & 37 & 3.67 & prognosis \\
3 & 27 & 5.97 & down regulation & 13 & 20 & 3.65 & lung cancer \\
4 & 9 & 4.92 & germline mutation & 14 & 34 & 3.60 & microRNA \\
5 & 21 & 4.91 & gene expression & 15 & 11 & 3.36 & microRNA expression \\
6 & 10 & 4.80 & microsatellite instability & 16 & 5 & 3.12 & pten/mmac1 gene \\
7 & 46 & 4.33 & cancer & 17 & 57 & 3.10 & invasion \\
8 & 21 & 4.33 & migration & 18 & 74 & 3.03 & gene \\
9 & 40 & 4.18 & activation & 19 & 8 & 3.01 & mTOR \\
10 & 10 & 3.90 & breast & 20 & 75 & 2.95 & apoptosis \\
\hline
\end{tabular}


Table 6: Main characteristics of eligible studies

\begin{tabular}{|c|c|c|c|c|c|c|c|c|c|}
\hline First author & Year & Country & Ethnicity & Antibody Source & Cases & Control & Risk to cancer & Outcome & Quality \\
\hline Badary DM & 2017 & Egypt & European & Labvision & 42 & & & Pos & 7 \\
\hline Kang HJ & 2016 & Korea & Asian & Cell signal & 272 & & & Pos & 8 \\
\hline Kim HS & 2016 & Korea & Asian & Cell signal & 438 & & & & 8 \\
\hline Zhang XL & 2015 & China & Asian & DAKO & 48 & & & Pos & 7 \\
\hline Tapia $\mathrm{O}$ & 2014 & Chile & ---- & Cell signal & 71 & & & & 7 \\
\hline $\mathrm{LiY}$ & 2013 & China & Asian & Cell signal & 114 & & & & 8 \\
\hline Zhu XH & 2013 & China & Asian & Cell signal & 159 & & & & 8 \\
\hline Li M & 2012 & China & Asian & Cell signal & 33 & & & & 7 \\
\hline Yang Z & 2013 & China & Asian & Abcam & 50 & 41 & Down & & 8 \\
\hline Bai ZG & 2007 & China & Asian & Zymed & 91 & & & Pos & 8 \\
\hline Zheng HC & 2006 & Japan & Asian & Novocastra & 249 & 49 & Down & Pos & 9 \\
\hline Deng $\mathrm{H}$ & 2006 & China & Asian & Cell signal & 118 & & & Pos & 8 \\
\hline Lm SA & 2005 & Korea & Asian & Neomarker & 84 & & & Pos & 8 \\
\hline Lee HS & 2003 & Korea & Asian & Fremont & 329 & & & Pos & 8 \\
\hline Zheng HC & 2003 & China & Asian & Zymed & 113 & 113 & Down & & 9 \\
\hline Yang L & 2003 & China & Asian & Zymed & 184 & 102 & Down & & 9 \\
\hline Li JY & 2004 & China & Asian & Zymed & 67 & 56 & Down & & 7 \\
\hline
\end{tabular}

Down, down-regulated expression; Pos, positive correlation.

tongue squamous cancer [52], head and neck cancer [53], pancreatic cancer [54], colorectal cancer [55], lung cancer [56], sacral chordoma [57], salivary adenoid cystic carcinoma [58], renal clear cell carcinoma [59], laryngeal and hypopharyngeal squamous cell carcinoma [60], esophageal cancer [61], we found that PTEN underexpression was detected in gastric cancer than mucosa or dysplasia, and negatively with depth of invasion, lymph node and distant metastasis, TNM staging, and dedifferentiation of gastric cancer according to meta-analysis, while versa for its mRNA level according to the bioinformatics analysis. Zhou et al. [62] demonstrated that the expression of PTEN mRNA and protein was significantly lower in hepatocellular carcinoma than the paracancerous tissues. The discrepancy might be due to different methodologies, a complex event from mRNA to protein, and a positive feedback overexpression of PTEN mRNA during progression. These results indicate that aberrant PTEN expression promotes gastric carcinogenesis and is considered as a good marker for aggressive behaviors of gastric cancer.

Reportedly, PTEN overexpression was associated with favorable prognosis in breast cancer [63], colorectal cancer [55], triple-negative breast cancer [64], lung cancer [56], ampullary adenocarcinoma [65], pancreatic cancer [66], gastrointestinal stromal tumor [67], oral squamous carcinoma [68], esophageal carcinoma [61], ovarian cancer [69], and hepatocellular carcinoma [70]. It might be also demonstrated to indicate the favorable prognosis of tongue squamous carcinoma [52], colorectal cancer [71], renal cell carcinoma [72], endometrial carcinoma [73], esophageal adenocarcinoma [74], mesothelioma [75], lung cancer [76], and invasive ductal carcinoma of the breast [77] as an independent factor. Our study showed that PTEN expression was positively linked to the favorable prognosis of the gastric cancer patients at either mRNA or protein level. da Costa et al. [78] demonstrated that PTEN hypoexpression was positively associated with a short overall survival of head and neck squamous cell carcinoma patients undergoing chemotherapy and cetuximab. Endoh et al. [79] found that PTEN expression was positively linked to a long survival after in EGFR-mutated lung cancer patients receiving gefitinib ademinstration. These findings suggest that PTEN loss is considered as a potential good marker for unfavorable prognosis of the gastric cancer patients at both mRNA and protein levels.

In conclusion, the study about PTEN and gastric cancer mainly focused on PTEN discovery, correlation of its genetic and epigenetic alteration with cancers, the effects of PTEN expression on the phenotypes of gastric cancer cells, and the regulatory effects of miRNA on PTEN translation. PTEN expression was down-regulated during gastric carcinogenesis as a late event, and was negatively associated with the aggressiveness and poor prognosis of gastric cancer at both mRNA and protein levels. The following disadvantages are mentioned in our study. Firstly, the positive results are published to cause 
Table 7: The prognostic significance of PTEN mRNA in gastric cancer

\begin{tabular}{|c|c|c|c|c|}
\hline \multirow{2}{*}{ Clinicopathological features } & \multicolumn{2}{|c|}{ Overall survival } & \multicolumn{2}{|c|}{ Progression-free survival } \\
\hline & Hazard ratio & $p$ & Hazard ratio & $p$ \\
\hline \multicolumn{5}{|l|}{ Sex } \\
\hline Female & $1.26(0.8-2)$ & 0.32 & $1.36(0.87-2.13)$ & 0.18 \\
\hline Male & $0.58(0.43-0.78)$ & $3 e-04$ & $0.63(0.47-0.84)$ & 0.0016 \\
\hline \multicolumn{5}{|l|}{$\mathrm{T}$} \\
\hline 2 & $0.65(0.42-1)$ & 0.05 & $0.66(0.43-1)$ & 0.047 \\
\hline 3 & $0.59(0.41-0.84)$ & 0.0037 & $0.66(0.47-0.93)$ & 0.017 \\
\hline 4 & $1.76(0.77-4)$ & 0.17 & $1.46(0.68-3.15)$ & 0.33 \\
\hline \multicolumn{5}{|l|}{$\mathrm{N}$} \\
\hline 0 & $1.49(0.54-4.1)$ & 0.44 & $1.59(0.58-4.35)$ & 0.36 \\
\hline $1-3$ & $0.61(0.47-0.8)$ & 0.00025 & $0.63(0.49-0.81)$ & 0.00035 \\
\hline 1 & $0.5(0.33-0.75)$ & 0.00067 & $0.52(0.35-0.77)$ & 0.00081 \\
\hline 2 & $0.46(0.29-0.72)$ & 0.00059 & $0.46(0.3-0.71)$ & 0.00038 \\
\hline 3 & $1.38(0.81-2.34)$ & 0.23 & $0.75(0.41-1.36)$ & 0.34 \\
\hline \multicolumn{5}{|l|}{ M } \\
\hline 0 & $0.67(0.5-0.88)$ & 0.0046 & $0.68(0.52-0.89)$ & 0.0043 \\
\hline 1 & $0.64(0.36-1.16)$ & 0.14 & $1.36(0.7-2.63)$ & 0.36 \\
\hline \multicolumn{5}{|l|}{ TNM staging } \\
\hline I & $0.29(0.08-1.06)$ & 0.047 & $0.31(0.09-1.13)$ & 0.062 \\
\hline II & $0.52(0.25-1.08)$ & 0.075 & $0.5(0.24-1.01)$ & 0.049 \\
\hline III & $0.6(0.41-0.88)$ & 0.0084 & $0.61(0.42-0.88)$ & 0.0084 \\
\hline IV & $0.61(0.41-0.93)$ & 0.019 & $0.74(0.5-1.1)$ & 0.14 \\
\hline \multicolumn{5}{|l|}{ Differentiation } \\
\hline Well-differentiated & - & - & - & - \\
\hline Moderately-differentiated & $0.4(0.19-0.84)$ & 0.012 & $0.43(0.21-0.85)$ & 0.013 \\
\hline Poorly-differentiated & $1.33(0.82-2.17)$ & 0.25 & $1.3(0.82-2.06)$ & 0.26 \\
\hline \multicolumn{5}{|l|}{ Lauren's classification } \\
\hline Intestinal-type & $0.38(0.26-0.55)$ & $1.2 \mathrm{e}-07$ & $0.47(0.33-0.67)$ & $2.4 \mathrm{e}-05$ \\
\hline Diffuse-type & $0.72(0.5-1.02)$ & 0.063 & $0.75(0.53-1.06)$ & 0.11 \\
\hline Mixed-type & $3.38(1.13-10.14)$ & 0.021 & $2.79(0.94-8.24)$ & 0.054 \\
\hline \multicolumn{5}{|l|}{ Her2 positivity } \\
\hline- & $0.75(0.56-1)$ & 0.047 & $0.78(0.58-1.04)$ & 0.094 \\
\hline+ & $0.57(0.38-0.84)$ & 0.0046 & $0.48(0.31-0.74)$ & 0.00076 \\
\hline \multicolumn{5}{|l|}{ Treatment } \\
\hline Surgery alone & $0.75(0.55-1.02)$ & 0.067 & $0.8(0.61-1.06)$ & 0.13 \\
\hline 5-FU-based adjuvant & $0.44(0.16-1.26)$ & 0.12 & $0.63(0.26-1.55)$ & 0.31 \\
\hline Other adjuvant & $1.57(0.63-3.93)$ & 0.33 & $0.71(0.32-1.56)$ & 0.39 \\
\hline
\end{tabular}

publication bias. Secondly, survival data were extracted from published Kaplan-Meier curves using software to cause processing bias. Thirdly, this small sample size influences the association strength between PTEN expression and clinicopathological characteristics to cause subject bias. Fourthly, bibliometric analysis calculates the co-words and co-citation, whose random property influences the final conclusion. Fifthly, bioinformatics analysis used the data of cDNA array and RNA sequencing of tissues, so cell type and proportion of tissues and the

disadvantages of both high-throughput methods affect the results' accuracy.

\section{MATERIALS AND METHODS}

\section{Identification of eligible studies and data extraction}

We performed a publication search using PubMed, Web of Science, BIOSIS SciFinder and CNKI 
updated on March 14, 2017. The following search terms were used: (PTEN OR MMAC1 OR TEP1) AND (gastric OR stomach) AND (cancer OR carcinoma OR adenocarcinoma). Searching was done without restriction on language or publication years. Inclusion criteria for studies: (1) articles to observe the alteration in PTEN expression in gastric cancer by immunohistochemistry; (2) papers to compare PTEN expression with pathobiological behaviors and prognosis of gastric cancer by immunohistochemistry. Exclusion criteria included: (1) abstract, comment, review and meeting; (2) duplication of the previous publications; (3) Western blot, RT-PCR, cDNA microarray, or transcriptomic sequencing for PTEN expression; (4) lack of sufficient information.

\section{Bibliometric analysis}

The downloaded files about PTEN from Web of Science was input into CiteSpace II (http://cluster.cis. drexel.edu/ cchen/citespace/), which is a freely available Java application for visualizing and analyzing trends and patterns in scientific literature. Firstly, we performed a hybrid network and timeline co-citation cluster analysis of the references with term labeled. After that, key terms were determined and subjected to co-word analysis.

\section{Data extraction}

Based on the inclusion criteria, two reviewers (HC Zheng and YH Qiu) independently extracted information from all eligible publications. The following information were included in each study: name of first author, year of publication, country, ethnicity, cancer types, source of control, antibody company, numbers of cases and controls, expression alteration, correlation with aggressive features, and follow-up times. Regarding survival analysis, we used Engauge Digitizer software to extract data from KaplanMeier curves and calculated the Hazard ratios (HR) and their corresponding 95\% confidence intervals (CI). Any disagreement was resolved through discussion until the two reviewers reached a consensus.

\section{Quality score assessment}

Two reviewers (HC Zheng and S Zhao) independently assessed the quality of the included studies according to Newcastle Ottawa Scale (NOS, http://www. ohri.ca/programs/clinical_epidemiology/oxford.asp).

The scale consists of three components related to sample selection, comparability and ascertainment of outcome.

\section{Bioinformatics analysis}

PTEN expression was analyzed using Oncomine (www.oncomine.org). We compared the differences in PTEN mRNA level between gastric normal tissue and cancer. The expression data (RNA-seqV2) and clinicopathological data of 392 gastric cancer patients were downloaded from the Cancer Genome Atlas (TCGA, https://cancergenome.nih.gov/) database by TCGA-assembler in $\mathrm{R}$ software. We integrated the raw data, analyzed PTEN expression in gastric cancer, and compared it with clinicopathological and prognostic data of the patients with gastric cancer. Additionally, the prognostic significance of PTEN mRNA was also analyzed using Kaplan-Meier plotter (http://kmplot.com).

\section{Statistics analysis}

HWE was evaluated using Chi-square test in control groups of each study. Strength of association between PTEN expression and cancer risk was assessed by odds ratios with $95 \%$ confidence intervals. Statistical significance of the pooled OR was determined by $\mathrm{Z}$ test. If there was no significant heterogeneity, the fixed effect model (Mantel-Haenszel method) would be employed. Otherwise, the random effect model (DerSimonian and Laird method) would be used excluding prognostic analysis. Heterogeneity effect was then quantified by $\mathrm{I}^{2}$ test, which was subdivided into low, moderate and high degrees of heterogeneity according to the cut-off values of $25 \%, 50 \%$ and $75 \%$ respectively. Publication bias was evaluated by funnel plot and quantified by Begg's test and Egger's test to assess funnel plot asymmetry. Metaanalyses were performed with Revman software 5.3 and data from TCGA database was dealt with SPSS 10.0 using student t test. $P<0.05$ was considered as statistically significant.

\section{ACKNOWLEDGMENTS AND FUNDING}

This study was supported by Liaoning BaiQianWan Talents Program, Outstanding Scientific Fund of Shengjing Hospital, Award for Liaoning Distinguished Professor, a Key Scientific and Technological Project of Liaoning Province (2015408001) and National Natural Scientific Foundation of China (81472544; 81672700).

\section{CONFLICTS OF INTEREST}

The authors have declared that no competing interests exist.

\section{REFERENCES}

1. Leslie NR, Kriplani N, Hermida MA, Alvarez-Garcia V, Wise HM. The PTEN protein: cellular localization and post-translational regulation. Biochem Soc Trans. 2016; 44:273-278.

2. Li A, Qiu M, Zhou H, Wang T, Guo W. PTEN, insulin resistance, and cancer. Curr Pharm Des. 2017; 23: 3667-3676. 
3. Phadngam S, Castiglioni A, Ferraresi A, Morani F, Follo C, Isidoro C. PTEN dephosphorylates AKT to prevent the expression of GLUT1 on plasmamembrane and to limit glucose consumption in cancer cells. Oncotarget. 2016; 7:84999-85020. https://doi.org/10.18632/oncotarget.13113.

4. Gu T, Zhang Z, Wang J, Guo J, Shen WH, Yin Y. CREB is a novel nuclear target of PTEN phosphatase. Cancer Res. 2011; 71:2821-2825.

5. Yang Z, Xie C, Xu W, Liu G, Cao X, Li W, Chen J, Zhu Y, Luo S, Luo Z, Lu N. Phosphorylation and inactivation of PTEN at residues Ser380/Thr382/383 induced by Helicobacter pylori promotes gastric epithelial cell survival through PI3K/Akt pathway. Oncotarget. 2015; 6:31916-31926. https://doi. org/10.18632/oncotarget.5577.

6. Torres J, Pulido R. The tumor suppressor PTEN is phosphorylated by the protein kinase CK2 at its $\mathrm{C}$ terminus. Implications for PTEN stability to proteasome-mediated degradation. J Biol Chem. 2001; 276:993-998.

7. Liang H, He S, Yang J, Jia X, Wang P, Chen X, Zhang Z, Zou $\mathrm{X}$, McNutt MA, Shen WH, Yin Y. PTENa, a PTEN isoform translated through alternative initiation, regulates mitochondrial function and energy metabolism. Cell Metab. 2014; 19:836-848.

8. Liang H, Chen X, Yin Q, Ruan D, Zhao X, Zhang C, McNutt MA, Yin Y. PTEN $\beta$ is an alternatively translated isoform of PTEN that regulates rDNA transcription. Nat Commun. 2017; 8:14771.

9. Benitez JA, Ma J, D'Antonio M, Boyer A, Camargo MF, Zanca C, Kelly S, Khodadadi-Jamayran A, Jameson NM, Andersen M, Miletic H, Saberi S, Frazer KA, et al. PTEN regulates glioblastoma oncogenesis through chromatinassociated complexes of DAXX and histone H3. Nat Commun. 2017; 8:15223.

10. Petrella BL, Brinckerhoff CE. PTEN suppression of YY1 induces HIF-2 activity in von-Hippel-Lindau-null renal-cell carcinoma. Cancer Biol Ther. 2009; 8:1389-1401.

11. Lee MS, Jeong MH, Lee HW, Han HJ, Ko A, Hewitt SM, Kim JH, Chun KH, Chung JY, Lee C, Cho H, Song J. PI3K/AKT activation induces PTEN ubiquitination and destabilization accelerating tumourigenesis. Nat Commun. 2015; 6:7769.

12. Yang JM, Schiapparelli P, Nguyen HN, Igarashi A, Zhang Q, Abbadi S, Amzel LM, Sesaki H, Quiñones-Hinojosa A, Iijima M. Characterization of PTEN mutations in brain cancer reveals that pten mono-ubiquitination promotes protein stability and nuclear localization. Oncogene. 2017; 36:3673-3685.

13. Guan J, Zhao Q, Mao W. Nuclear PTEN interferes with binding of $\mathrm{Ku} 70$ at double- strand breaks through posttranslational poly (ADP-ribosyl)ation. Biochim Biophys Acta. 2016; 1863:3106-3115.

14. Wang X, Trotman LC, Koppie T, Alimonti A, Chen Z, Gao Z, Wang J, Erdjument-Bromage H, Tempst P, Cordon-Cardo C, Pandolfi PP, Jiang X. NEDD4-1 is a proto-oncogenic ubiquitin ligase for PTEN. Cell. 2007; 128:129-139.
15. Shao C, Li Z, Ahmad N, Liu X. Regulation of PTEN degradation and NEDD4-1 E3 ligase activity by Numb. Cell Cycle. 2017; 16:957-967.

16. Maddika S, Kavela S, Rani N, Palicharla VR, Pokorny JL, Sarkaria JN, Chen J. WWP2 is an E3 ubiquitin ligase for PTEN. Nat Cell Biol. 2011; 13:728-733.

17. Ahn Y, Hwang CY, Lee SR, Kwon KS, Lee C. The tumour suppressor PTEN mediates a negative regulation of the E3 ubiquitin-protein ligase Nedd4. Biochem J. 2008; 412:331338.

18. Zhang J, Zhang P, Wei Y, Piao HL, Wang W, Maddika S, Wang M, Chen D, Sun Y, Hung MC, Chen J, Ma L. Deubiquitylation and stabilization of PTEN by USP13. Nat Cell Biol. 2013; 15:1486-1494.

19. Lee JT, Shan J, Zhong J, Li M, Zhou B, Zhou A, Parsons R, $\mathrm{Gu}$ W. RFP-mediated ubiquitination of PTEN modulates its effect on AKT activation. Cell Res. 2013; 23:552-564.

20. Meng Z, Jia LF, Gan YH. PTEN activation through K163 acetylation by inhibiting HDAC6 contributes to tumor inhibition. Oncogene. 2016; 35:2333-2344.

21. Knobbe CB, Lapin V, Suzuki A, Mak TW. The roles of PTEN in development, physiology and tumorigenesis in mouse models:a tissue-by-tissue survey. Oncogene. 2008; 27:5398-5415.

22. Li JY, Zheng HC, Yang L, Xu L, Yang XF, Gao H, Zhang $\mathrm{YC}$, Xin Y. [Altered expression of PTEN gene and $\mathrm{LOH}$ of its epigenetic microsatellite in gastric carcinoma]. [Article in Chinese]. Zhonghua Zhong Liu Za Zhi. 2004; 26:389-392.

23. Yang Z, Yuan XG, Chen J, Luo SW, Luo ZJ, Lu NH. Reduced expression of PTEN and increased PTEN phosphorylation at residue Ser380 in gastric cancer tissues:a novel mechanism of PTEN inactivation. Clin Res Hepatol Gastroenterol. 2013; 37:72-79.

24. Yang L, Kuang LG, Zheng HC, Li JY, Wu DY, Zhang SM, Xin Y. PTEN encoding product: a marker for tumorigenesis and progression of gastric carcinoma. World J Gastroenterol. 2003; 9:35-39.

25. Zheng H, Takahashi H, Murai Y, Cui Z, Nomoto K, Tsuneyama K, Takano Y. Low expression of FHIT and PTEN correlates with malignancy of gastric carcinomas: tissue-array findings. Appl Immunohistochem Mol Morphol. 2007; 15:432-440.

26. Zheng HC, Sun JM, Li XH, Yang XF, Zhang YC, Xin Y. Role of PTEN and MMP-7 expression in growth, invasion, metastasis and angiogenesis of gastric carcinoma. Pathol Int. 2003; 53:659-666.

27. Badary DM, Abdel-Wanis ME, Hafez MZ, Aboulhagag NA. Immunohistochemical analysis of PTEN, HER2/neu, and ki67 expression in patients with gastric cancer and their association with survival. Pathophysiology. 2017; 24:99106.

28. Bai Z, Ye Y, Chen D, Shen D, Xu F, Cui Z, Wang S. Homeoprotein $\mathrm{Cdx} 2$ and nuclear PTEN expression profiles are related to gastric cancer prognosis. APMIS. 2007; 115:1383-1390. 
29. Deng H, Wu RL, Zhou HY, Huang X, Chen Y, Liu LJ. Significance of Survivin and PTEN expression in full lymph node-examined gastric cancer. World J Gastroenterol. 2006; 12:1013-1017.

30. Kang HJ, Lee IS, Park YS, Ho WJ, Sohn D, Ahn JY, Yook JH, Kim BS. Biomarkers of EBV-positive Gastric Cancers: Loss of PTEN Expression is Associated with Poor Prognosis and Nodal Metastasis. Ann Surg Oncol. 2016; 23:3684-3692.

31. Kim HS, Shin SJ, Beom SH, Jung M, Choi YY, Son T, Kim HI, Cheong JH, Hyung WJ, Noh SH, Chung H, Park JC, Shin SK, et al. Comprehensive expression profiles of gastric cancer molecular subtypes by immunohistochemistry: implications for individualized therapy. Oncotarget. 2016; 7:44608-44620. https://doi.org/10.18632/oncotarget.10115.

32. Li M, Sun H, Song L, Gao X, Chang W, Qin X. Immunohistochemical expression of mTOR negatively correlates with PTEN expression in gastric carcinoma. Oncol Lett. 2012; 4:1213-1218.

33. Li Y, Cui J, Zhang CH, Yang DJ, Chen JH, Zan WH, Li B, Li Z, He YL. High-expression of DJ-1 and loss of PTEN associated with tumor metastasis and correlated with poor prognosis of gastric carcinoma. Int J Med Sci. 2013; 10:1689-1697.

34. Zhang X, Park JS, Park KH, Kim KH, Jung M, Chung HC, Rha SY, Kim HS. PTEN deficiency as a predictive biomarker of resistance to HER2-targeted therapy in advanced gastric cancer. Oncology. 2015; 88:76-85.

35. Zhu X, Qin X, Fei M, Hou W, Greshock J, Bachman KE, Kang J, Qin CY. Loss and reduced expression of PTEN correlate with advanced-stage gastric carcinoma. Exp Ther Med. 2013; 5:57-64.

36. Im SA, Lee KE, Nam E, Kim DY, Lee JH, Han HS, Seoh JY, Park HY, Cho MS, Han WS, Lee SN. Potential prognostic significance of p185 (HER2) overexpression with loss of PTEN expression in gastric carcinomas. Tumori. 2005; 91:513-521.

37. Tapia O, Riquelme I, Leal P, Sandoval A, Aedo S, Weber H, Letelier P, Bellolio E, Villaseca M, Garcia P, Roa JC. The PI3K/AKT/mTOR pathway is activated in gastric cancer with potential prognostic and predictive significance. Virchows Arch. 2014; 465:25-33.

38. Lee HS, Lee HK, Kim HS, Yang HK, Kim WH. Tumor suppressor gene expression correlates with gastric cancer prognosis. J Pathol. 2003; 200:39-46.

39. Kim SJ, Lee HW, Baek JH, Cho YH, Kang HG, Jeong JS, Song J, Park HS, Chun KH. Activation of nuclear PTEN by inhibition of Notch signaling induces G2/M cell cycle arrest in gastric cancer. Oncogene. 2016; 35:251-260.

40. Xie S, Lu Z, Lin Y, Shen L, Yin C. Upregulation of PTEN suppresses invasion in Tca8113 tongue cancer cells through repression of epithelial-mesenchymal transition (EMT). Tumor Biol. 2016; 37:6681-6689.

41. Zhang LL, Mu GG, Ding QS, Li YX, Shi YB, Dai JF, Yu HG. Phosphatase and Tensin Homolog (PTEN) Represses
Colon Cancer Progression through Inhibiting Paxillin Transcription via PI3K/AKT/NF-кB Pathway. J Biol Chem. 2015; 290:15018-15029.

42. Hu Y, Xu S, Jin W, Yi Q, Wei W. Effect of the PTEN gene on adhesion, invasion and metastasis of osteosarcoma cells. Oncol Rep. 2014; 32:1741-1747.

43. Zhang LL, Liu J, Lei S, Zhang J, Zhou W, Yu HG. PTEN inhibits the invasion and metastasis of gastric cancer via downregulation of FAK expression. Cell Signal. 2014; 26:1011-1020.

44. Vitolo MI, Boggs AE, Whipple RA, Yoon JR, Thompson K, Matrone MA, Cho EH, Balzer EM, Martin SS. Loss of PTEN induces microtentacles through PI3K-independent activation of cofilin. Oncogene. 2013; 32:2200-2210.

45. Park MJ, Kim MS, Park IC, Kang HS, Yoo H, Park SH, Rhee CH, Hong SI, Lee SH. PTEN suppresses hyaluronic acid-induced matrix metalloproteinase-9 expression in U87MG glioblastoma cells through focal adhesion kinase dephosphorylation. Cancer Res. 2002; 62:6318-6322.

46. Chung JH, Ostrowski MC, Romigh T, Minaguchi T, Waite $\mathrm{KA}$, Eng C. The ERK1/2 pathway modulates nuclear PTENmediated cell cycle arrest by cyclin D1 transcriptional regulation. Hum Mol Genet. 2006; 15:2553-2559.

47. Chen JH, Zhang P, Chen WD, Li DD, Wu XQ, Deng R, Jiao L, Li X, Ji J, Feng GK, Zeng YX, Jiang JW, Zhu XF. ATMmediated PTEN phosphorylation promotes PTEN nuclear translocation and autophagy in response to DNA-damaging agents in cancer cells. Autophagy. 2015; 11:239-252.

48. Errafiy R, Aguado C, Ghislat G, Esteve JM, Gil A, Loutfi $\mathrm{M}$, Knecht E. PTEN increases autophagy and inhibits the ubiquitin-proteasome pathway in glioma cells independently of its lipid phosphatase activity. PLoS One. 2013; 8:e83318.

49. Arico S, Petiot A, Bauvy C, Dubbelhuis PF, Meijer AJ, Codogno P, Ogier-Denis E. The tumor suppressor PTEN positively regulates macroautophagy by inhibiting the phosphatidylinositol 3-kinase/protein kinase B pathway. J Biol Chem. 2001; 276:35243-35246.

50. Zhao GF, Zhao S, Liu JJ, Wu JC, He HY, Ding XQ, Yu XW, Huang KQ, Li ZJ, Zheng HC. Cytokeratin 19 promoter directs the expression of Cre recombinase in various epithelia of transgenic mice. Oncotarget. 2017; 8:1830318311. https://doi.org/10.18632/oncotarget.15435.

51. Jang KS, Song YS, Jang SH, Min KW, Na W, Jang SM, Jun YJ, Lee KH, Choi D, Paik SS. Clinicopathological significance of nuclear PTEN expression in colorectal adenocarcinoma. Histopathology. 2010; 56:229-239.

52. Zhao J, Chi J, Gao M, Zhi J, Li Y, Zheng X. Loss of PTEN expression is associated with high microRNA 24 level and poor prognosis in patients with tongue squamous cell carcinoma. J Oral Maxillofac Surg. 2017; 75:1449.e1-1449.e8.

53. Ahmed MW, Kayani MA, Shabbir G, Ali SM, Shinwari WU, Mahjabeen I. Expression of PTEN and its correlation with proliferation marker Ki-67 in head and neck cancer. Int J Biol Markers. 2016; 31:193-203. 
54. Huang W, Yang J, Ren J, Tang J. Expression of PTEN and KAI1 tumor suppressor genes in pancreatic carcinoma and its association with different pathological factors. Oncol Lett. 2016; 11:559-562.

55. Li XH, Zheng HC, Takahashi H, Masuda S, Yang XH, Takano Y. PTEN expression and mutation in colorectal carcinomas. Oncol Rep. 2009; 22:757-764.

56. Wang J, Chen H, Liao Y, Chen N, Liu T, Zhang H, Zhang $\mathrm{H}$. Expression and clinical evidence of miR-494 and PTEN in non-small cell lung cancer. Tumor Biol. 2015; 36:69656972.

57. Chen K, Mo J, Zhou M, Wang G, Wu G, Chen H, Zhang $\mathrm{K}$, Yang H. Expression of PTEN and mTOR in sacral chordoma and association with poor prognosis. Med Oncol. 2014; 31:886.

58. Chen D, Zhang B, Kang J, Ma X, Lu Y, Gong L. Expression and clinical significance of FAK, ILK, and PTEN in salivary adenoid cystic carcinoma. Acta Otolaryngol. 2013; 133:203-208.

59. Cheng T, Zhang JG, Cheng YH, Gao ZW, Ren XQ. Relationship between PTEN and Livin expression and malignancy of renal cell carcinomas. Asian Pac J Cancer Prev. 2012; 13:2681-2685.

60. Liu J, Lei DP, Jin T, Zhao XN, Li G, Pan XL. Altered expression of miR-21 and PTEN in human laryngeal and hypopharyngeal squamous cell carcinomas. Asian Pac J Cancer Prev. 2011; 12:2653-2657.

61. Chang MS, Lee HS, Lee BL, Kim YT, Lee JS, Kim WH. Differential protein expression between esophageal squamous cell carcinoma and dysplasia, and prognostic significance of protein markers. Pathol Res Pract. 2005; 201:417-425.

62. Zhou X, Zhu H, Lu J. PTEN and hTERT gene expression and the correlation with human hepatocellular carcinoma. Pathol Res Pract. 2015; 211:316-319.

63. Golmohammadi R, Rakhshani MH, Moslem AR, Pejhan A. Prognostic Role of PTEN Gene Expression in Breast Cancer Patients from North-East Iran. Asian Pac J Cancer Prev. 2016; 17:4527-4531.

64. Beg S, Siraj AK, Prabhakaran S, Jehan Z, Ajarim D, Al-Dayel F, Tulbah A, Al-Kuraya KS. Loss of PTEN expression is associated with aggressive behavior and poor prognosis in Middle Eastern triple-negative breast cancer. Breast Cancer Res Treat. 2015; 151:541-553.

65. Shroff S, Overman MJ, Rashid A, Shroff RT, Wang H, Chatterjee D, Katz MH, Lee JE, Wolff RA, Abbruzzese JL, Fleming JB, Wang H. The expression of PTEN is associated with improved prognosis in patients with ampullary adenocarcinoma after pancreatico- duodenectomy. Arch Pathol Lab Med. 2013; 137:1619-1626.

66. Feng C, Yao R, Huang F, Liu X, Nie W. High level of PTEN expression is associated with low-grade liver metastasis and satisfactory patient survival in pancreatic cancer. Arch Med Res. 2011; 42:584-588.
67. Zhang Y, Yu D, Li X, Hu J, Gong J. Reduced expression of PTEN protein and its prognostic significance in the gastrointestinal stromal tumor. J Huazhong Univ Sci Technolog Med Sci. 2010; 30:165-169.

68. Kurasawa Y, Shiiba M, Nakamura M, Fushimi K, Ishigami T, Bukawa H, Yokoe H, Uzawa K, Tanzawa H. PTEN expression and methylation status in oral squamous cell carcinoma. Oncol Rep. 2008; 19:1429-1434.

69. Sui L, Dong Y, Watanabe Y, Yamaguchi F, Sugimoto K, Tokuda M. Alteration and clinical relevance of PTEN expression and its correlation with survivin expression in epithelial ovarian tumors. Oncol Rep. 2006; 15:773-778.

70. $\mathrm{Hu} \mathrm{TH}$, Wang $\mathrm{CC}$, Huang $\mathrm{CC}$, Chen $\mathrm{CL}$, Hung $\mathrm{CH}$, Chen $\mathrm{CH}$, Wang JH, Lu SN, Lee CM, Changchien CS, Tai MH. Down-regulation of tumor suppressor gene PTEN, overexpression of p53, plus high proliferating cell nuclear antigen index predict poor patient outcome of hepatocellular carcinoma after resection. Oncol Rep. 2007; 18:1417-1426.

71. Yazdani Y, Farazmandfar T, Azadeh H, Zekavatian Z. The prognostic effect of PTEN expression status in colorectal cancer development and evaluation of factors affecting it: miR-21 and promoter methylation. J Biomed Sci. 2016; 23:9.

72. Zhu C, Wei J, Tian X, Li Y, Li X. Prognostic role of PPAR- $\gamma$ and PTEN in the renal cell carcinoma. Int J Clin Exp Pathol. 2015; 8:12668-12677.

73. Akiyama-Abe A, Minaguchi T, Nakamura Y, Michikami H, Shikama A, Nakao S, Sakurai M, Ochi H, Onuki M, Matsumoto K, Satoh T, Oki A, Yoshikawa H. Loss of PTEN expression is an independent predictor of favourable survival in endometrial carcinomas. Br J Cancer. 2013; 109:1703-1710.

74. Bettstetter M, Berezowska S, Keller G, Walch A, Feuchtinger A, Slotta-Huspenina J, Feith M, Drecoll E, Höfler H, Langer R. Epidermal growth factor receptor, phosphatidylinositol-3-kinase catalytic subunit/PTEN, and KRAS/NRAS/BRAF in primary resected esophageal adenocarcinomas: loss of PTEN is associated with worse clinical outcome. Hum Pathol. 2013; 44:829-836.

75. Opitz I, Soltermann A, Abaecherli M, Hinterberger M, Probst-Hensch N, Stahel R, Moch H, Weder W. PTEN expression is a strong predictor of survival in mesothelioma patients. Eur J Cardiothorac Surg. 2008; 33:502-506.

76. Tang JM, He QY, Guo RX, Chang XJ. Phosphorylated Akt overexpression and loss of PTEN expression in non-small cell lung cancer confers poor prognosis. Lung Cancer. 2006; 51:181-191.

77. Lee JS, Kim HS, Kim YB, Lee MC, Park CS, Min KW. Reduced PTEN expression is associated with poor outcome and angiogenesis in invasive ductal carcinoma of the breast. Appl Immunohistochem Mol Morphol. 2004; 12:205-210.

78. da Costa AA, D'Almeida Costa F, Ribeiro AR, Guimarães AP, Chinen LT, Lopes CA, de Lima VC. Low PTEN expression is associated with worse overall survival in head and neck squamous cell carcinoma patients treated 
with chemotherapy and cetuximab. Int J Clin Oncol. 2015; 20:282-289.

79. Endoh H, Yatabe Y, Kosaka T, Kuwano H, Mitsudomi T. PTEN and PIK3CA expression is associated with prolonged survival after gefitinib treatment in EGFR-mutated lung cancer patients. J Thorac Oncol. 2006; 1:629-634. 